\title{
Análise bibliométrica de Trabalhos de Conclusão de um Curso de Odontologia no Nordeste brasileiro
}

\author{
Bruno Dias Florêncio Leite*; Taiana Brito Menêzes**; Luiz Roberto Augusto Noro***
}

\section{RESUMO}

O objetivo do presente estudo foi identificar a tendência da produção científica de um curso de Odontologia, a partir dos Trabalhos de Conclusão de Curso (TCC), os quais consistem no desenvolvimento de pesquisa por aluno de graduação, baseado na necessidade da produção de conhecimento e educação permanente. Trata-se de uma pesquisa bibliométrica por meio de estudo transversal, de caráter observacional descritivo, com abordagem quantitativa. Observou-se o predomínio de estudos seccionais, desenvolvidos com seres humanos como sujeitos da pesquisa, com enfoque na área clínica, sendo $82 \%$ realizados com dados primários. Do total, $62 \%$ foram submetidos ao comitê de ética em pesquisa institucional. Observou-se que das referências analisadas, $81 \%$ eram artigos em periódicos, $11 \%$ livros, $3 \%$ teses e dissertações e $5 \%$ outros documentos, em especial de sites ou publicações oficiais. O TCC deve ser compreendido como conquista fundamental para estimular a criatividade, a educação permanente e a busca dos alunos por conhecimento a partir da pesquisa e não uma mera tarefa exigida pelo Projeto Pedagógico. Seria importante, também, enfatizar a construção de projetos de pesquisa coerentes com os princípios das Diretrizes Curriculares Nacionais dos cursos de Odontologia, em
Cirurgião-dentista pela UFRN

* Mestre em Saúde Coletiva, Programa de PósGraduação em Saúde Coletiva da UFRN

*** Professor do Programa de Pós-Graduação em Saúde Coletiva e do Departamento de Odontologia da UFRN

especial com estudos que pudessem se comprometer com avanços no Sistema Único de Saúde. O perfil da produção científica do curso de odontologia da UFRN realizada para o TCC é majoritariamente de estudos seccionais sobre temas de áreas clínicas, com coleta de dados primários, os quais são obtidos nas próprias dependências do curso. A maioria dos projetos é submetida ao CEP e utiliza artigos científicos como referências.

Descritores: Bibliometria. Indicadores de Produção Científica. Odontologia

\section{INTRODUÇÃO}

A abordagem pedagógica centrada fortemente na transmissão de conteúdos científicos, legitimada por professores com extremo conhecimento em sua área de atuação profissional ou acadêmica, tem demonstrado sua limitada contribuição para a formação exigida atualmente para a área da saúde.

A demanda social cada vez mais intensa por profissionais respaldados por valores morais, éticos, de cidadania e implicados com a transformação da sociedade em que atuam tem conduzido a universidade a repensar o perfil de seu egresso ${ }^{1}$. 
A formação superior em odontologia no Brasil, historicamente, esteve altamente alinhada aos interesses de mercado, definindo tanto a organização curricular quanto o perfil dos docentes, o que retardou a incorporação da pesquisa e da produção de conhecimento como parte do processo educativo ${ }^{2}$. A opção de valorizar e incrementar o processo de pesquisa na graduação tem como ponto de partida o entendimento de que a mesma pode ser tomada como princípio científico e educativo ${ }^{3}$.

O desenvolvimento de um projeto de pesquisa deve contribuir para que o aluno possa construir competências e habilidades na perspectiva da atualização constante, devendo ser capaz de buscar conhecimento novo, por meio de análise crítica da literatura, o que deve impactar positivamente em sua vida profissional futura ${ }^{4}$.

Buscando implantar esta concepção nos cursos de Odontologia, as Diretrizes Curriculares Nacionais (DCN) preveem como obrigatória a elaboração de um Trabalho de Conclusão de Curso (TCC) orientado por docente $^{5}$. A obrigatoriedade da pesquisa incentiva o aluno a utilizar a metodologia científica como ferramenta para resolução dos problemas, permitindo o desenvolvimento da criatividade e a continuidade dos estudos ${ }^{6}$. A compreensão e valorização do TCC mostra a importância da iniciação científica para o aguçamento da visão crítica, além de refinar a capacidade de trabalhar com orientação e em grupo.

Segundo alguns autores ${ }^{7}$ é necessário conhecer a atual realidade em pesquisas odontológicas e seu possível caminho para o desenvolvimento futuro. A pesquisa odontológica no Brasil experimentou um aumento quantitativo significativo nos primeiros anos do século $\mathrm{XXI}^{8,9}$, expresso pela maior divulgação em revistas especializadas, assim como a criação do Portal de Periódicos da Coordenação de Aperfeiçoamento de Pessoal de Nível Superior (CAPES), o qual permite consultar milhares de periódicos, com acesso rápido à produção científica mundial de forma atualizada $^{10}$. Para isto, as análises bibliométricas têm sido realizadas com o propósito de observar como têm sido conduzidas as pesquisas científicas, bem como identificar os temas de maior destaque e as inovações ocorridas ao longo do tempo ${ }^{9}$.

O TCC foi incluído na estrutura curricular do Curso de Odontologia da Universidade Federal do Rio Grande do Norte (UFRN) a partir do ano de 2007, sendo ofertado na lógica de duas atividades integradoras de formação: o TCC I ( $7^{\circ}$ período do curso), no qual os alunos desenvolvem o projeto de pesquisa e o TCC II ( $9^{\circ}$ período do curso), no qual os alunos apresentam, para uma banca de três professores, o TCC desenvolvido a partir do projeto previamente construído. O TCC tem, obrigatoriamente, a orientação de um professor.

O presente estudo teve por objetivo identificar a tendência da produção científica do curso de odontologia da UFRN por meio dos projetos de pesquisa dos TCC.

\section{MÉTODO}

O presente estudo diz respeito a uma pesquisa bibliométrica e trata-se de um estudo transversal, de caráter observacional descritivo, com abordagem quantitativa. $\mathrm{O}$ estudo caracteriza-se como transversal, pois os fatores em estudo e os possíveis efeitos são medidos em um único momento, e observacional descritivo, uma vez que descreve a ocorrência de uma situação, sem intervir sobre o objeto.

A bibliometria, por sua vez, busca orientar e organizar as informações obtidas no contexto científico-acadêmico, o que permite mapear e gerar diferentes indicadores de tratamento e gestão da informação e do conhecimento $^{11}$. Estudos bibliométricos surgiram devido à necessidade de estudar e avaliar as atividades de produção e 
comunicação científica, buscando também benefícios práticos e imediatos para as bibliotecas $^{12}$.

$\mathrm{O}$ material investigado consiste de projetos de pesquisa dos TCC do curso de Odontologia da UFRN, dos anos de 2011 a 2013. Os dados foram coletados por meio de formulário desenvolvido especificamente para este protocolo de pesquisa, contendo as seguintes informações: título do trabalho, tema do trabalho, área de atuação, tipo de estudo, tipo de fonte para coleta de dados, cenário onde foi realizado o trabalho, submissão a comitê de ética em pesquisa (CEP) e o tipo de referências utilizadas.

A coleta de dados foi feita exclusivamente pelo pesquisador principal, após definição compartilhada com o grupo de pesquisa sobre os critérios adotados para consideração das situações observadas.

Os projetos dos TCC foram obtidos junto à coordenação do Curso de Odontologia da UFRN. Mediante o acesso aos trabalhos, foi realizada a leitura dos resumos e foram analisadas as referências bibliográficas para a obtenção dos dados necessários ao preenchimento do formulário. Os dados foram tabulados com o auxílio do programa Microsoft Excel $2010 \AA$, sendo analisados por meio de estatística descritiva.

Tendo em vista a natureza do objeto da pesquisa, considerada como de domínio público, não foi necessária apreciação deste protocolo por Comitê de Ética em Pesquisa.

\section{RESULTADOS E DISCUSSÃO}

A partir da análise dos 38 projetos de TCC encontrados para o período em análise, foi realizada a extração das variáveis propostas para este estudo (tema do trabalho, área de atuação, tipo de estudo, tipo de fonte para coleta de dados, cenário proposto para trabalho, submissão ao CEP e o tipo de referências utilizadas), cujos resultados e discussões são apresentados nas seções seguintes. Não foram considerados os TCC classificados como relato de caso clínico ou revisão de literatura.

\section{Área de conhecimento e enfoque da pesquisa}

Para a variável "tema do trabalho", verificou-se maior concentração de pesquisas propostas nas áreas de Patologia Oral (18\%), Saúde Coletiva (11\%), Epidemiologia (11\%) e Cirurgia (11\%), que juntas totalizaram $50 \%$ dos projetos analisados (Tabela 1).

Um fator importante a ser observado é que as áreas mais pesquisadas são estudadas em períodos iniciais do curso de Odontologia, o qual é composto por nove semestres para o curso em estudo. Tendo em vista que o projeto de pesquisa é elaborado ao longo do sétimo semestre, existe dificuldade natural de contemplar conteúdos concomitantes ou posteriores a ele.

Realizou-se, também, uma análise quanto aos professores orientadores dos TCC e o componente curricular dos mesmos, observando-se que os professores mais envolvidos nas pesquisas foram também os professores da Patologia (oito trabalhos produzidos), seguidos novamente pela Saúde Coletiva e Cirurgia (seis trabalhos cada). Um dos fatores que pode contribuir com este resultado é a presença da pós-graduação stricto sensu na instituição, a qual abriga, inclusive na mesma estrutura física do curso de odontologia, programas nas áreas de Patologia Oral e Saúde Coletiva.

A presença de pós-graduandos e professores envolvidos nestes cursos de pósgraduação possivelmente oportuniza aos alunos da graduação bolsas de iniciação científica, implicando em um atrativo, além da possibilidade de atuação na área, tendo como provável produto a elaboração de projetos de pesquisa de TCC nas áreas de interesse da pósgraduação.

A exigência do TCC a partir das DCN 
Tabela 1 - Distribuição de projetos segundo área de conhecimento no Curso de Odontologia da UFRN nos anos de 2011 a 2013.

\begin{tabular}{l|cc}
\hline $\begin{array}{c}\text { Área } \\
\text { conhecimento }\end{array}$ & Total & \% \\
\hline Patologia Oral & 7 & 18 \\
Saúde Coletiva & 4 & 11 \\
Epidemiologia & 4 & 11 \\
Cirurgia & 4 & 11 \\
Endodontia & 3 & 7 \\
Materiais Dentários & 3 & 7 \\
Implantodontia & 2 & 5 \\
Estomatologia & 2 & 5 \\
DTM & 2 & 5 \\
Dentística & 2 & 5 \\
Periodontia & 1 & 3 \\
Prótese Dentária & 1 & 3 \\
Ortodontia & 1 & 3 \\
Microbiologia & 1 & 3 \\
Imagenologia & 1 & 3 \\
\hline
\end{tabular}

para o curso tem demandado esforços das universidades para que os trabalhos produzidos não se resumam a um mero exercício acadêmico, mas que possam constituir um indicador de qualidade e institucional, com possibilidade de gerar contribuições para resolução de problemas sociais ${ }^{13}$.

Considerando-se que os trabalhos podem ter enfoques nas áreas clínicas ou na saúde coletiva, pode-se observar um enfoque clínico predominante, em torno de cinco vezes maior que o na saúde coletiva. Este fato muito pode ser atribuído à conformação histórica da profissão e ao modelo ensino na saúde adotado por muito tempo nos cursos de saúde, entre eles o de odontologia.

No século XX, a formação dos profissionais de saúde assim como a estruturação dos sistemas públicos de diversos países, sofreu profundas influências do modelo norte-americano, conhecido como modelo flexneriano devido à publicação de um relatório sobre o ensino da medicina nos Estados Unidos por Abraham Flexner. Atualmente, o termoflexneriano é utilizado para fazer referência à medicina orientada para o indivíduo e também ao biologicismo, ao mecanicismo, à especialização e à tecnificação excessiva do trabalho em saúde, tratando-se de um modelo organizado em torno do hospitalescola como laboratório para as práticas profissionais, que trouxe consequências para a formação em saúde, tornando-se o paradigma dominante para explicar os fenômenos do processo saúde-doença ${ }^{14}$.

Alguns autores ${ }^{15}$ consideraram este modelo pedagógico centrado na perspectiva biologicista, medicalizante e procedimento centrada, ainda hegemônico, o que privilegia conteúdos, compartimentalização e dissociação da formação básica frente a formação profissionalizante e a fragmentação da formação em especialidades clínicas, além do hospital escola como única oportunidade de Revista da ABENO • 15(3):16-25, 2015. 
exercício da prática clínica.

A Lei de Diretrizes e Bases da Educação Brasileira e a implantação das DCN para os cursos de graduação em saúde foram importantes marcos para a promoção de mudanças no ensino superior no país, influenciando a adoção de estratégias para superação do modelo hegemônico, com especial ênfase a formação de profissionais aptos para atuação no sistema de saúde do país. As DCN para o curso de odontologia preconizam a formação de um profissional generalista, humanista, crítico e reflexivo, para atuar em todos os níveis de atenção à saúde, com base no rigor técnico e científico ${ }^{5}$. A referência explícita da formação voltada para o SUS está no item XXIX das habilidades específicas - no qual se afirma que o curso deve capacitar o aluno para "planejar e administrar serviços de saúde comunitária" - e no parágrafo único do artigo $5^{\circ}$, que define suas habilidades e estabelece que a formação do cirurgião-dentista deverá contemplar o sistema de saúde, a atenção integral da saúde num sistema regionalizado e hierarquizado de referência e contrarreferência e o trabalho em equipe" 5 .

É evidente a importância do conceito de saúde definido pela Constituição de 1988 e dos princípios de criação do SUS para a implantação das DCN, bem como o papel estratégico dessas DCN para o desenvolvimento do SUS ${ }^{16}$. Porém, todas essas questões não tiveram força para definir - de maneira explícita - que a formação odontológica no país deva ser voltada para a formação de um profissional capacitado para trabalhar no sistema público vigente no Brasil.

$\mathrm{O}$ perfil presente nas DCN recupera o perfil profissional definido pelo então Conselho Federal de Educação em 1962, de um dentista "geral, policlínico, destinado à coletividade ${ }^{17 "}$. Alguns autores ${ }^{18}$ consideram que, independentemente destas formulações, sempre se pretendeu a formação de um clínico geral, capaz de atender os problemas odontológicos da comunidade, surgindo desde este tempo até atualidade questões relativas à clínica integrada, multiprofissionalidade e atualização técnico-científica. No presente estudo este desafio ainda parece estar distante da realidade da graduação em Odontologia da UFRN.

A partir da visão de alunos concludentes de Odontologia no Ceará, a Saúde Bucal Coletiva possui conteúdos importantes abordados nas disciplinas que compõem a área, de abordagem pedagógica reconhecida como adequada, entretanto entre estes alunos foi destacado um maior interesse nas atividades clínicas tradicionais, mesmo quando pensa em sua inserção no SUS, possivelmente estimulada pela grande quantidade de disciplinas da área clínica nos cursos de Odontologia ${ }^{19}$.

Estudo realizado entre formandos do curso de Odontologia da Universidade Estadual de Ponta Grossa (PR) acerca de sua percepção quanto ao atual projeto pedagógico da instituição identificou grande valorização das disciplinas clínicas em relação às práticas de estágio no SUS, atividades de extensão e pesquisa, ou ao TCC. O estudo evidencia ainda que, apesar da percepção positiva da comunidade acadêmica, existem algumas fragilidades no atual projeto pedagógico do curso, as quais apontam para a necessidade de se avançar na construção de um currículo integrado e da consolidação da utilização de metodologias ativas no processo pedagógico ${ }^{20}$.

\section{Coleta de dados}

Os cenários para a realização dos TCC variam bastante de acordo com o tipo de estudo a ser realizado e, por isso, acaba ocorrendo em espaços físicos distintos. Observou-se que em sua maioria $(42 \%)$ os trabalhos se propuseram a realizar pesquisas nas próprias clínicas do Departamento de Odontologia, seguido do cenário laboratorial $(24 \%)$ e dos serviços de 
saúde (16\%). Observou-se ainda a existência de trabalhos propostos em espaços públicos, escolas, instituições de longa permanência, consultórios odontológicos, entre outros.

Os resultados encontrados para o local de estudo predominante podem ser explicados pela facilidade logística, como a disponibilidade de recursos e a participação dos professores orientadores na pesquisa. Além disso, observa-se também que há coerência entre o enfoque dos estudos e o cenário onde está sendo proposta a coleta de dados. A existência de propostas de trabalho em locais extramuros nos mostra a possibilidade da realização da pesquisa em campo, apesar do curto espaço de tempo disponível para a redação dos TCC.

Quanto à fonte de coleta de dados esta foi, em sua maioria, realizada por meio de dados primários (82\%), conforme observado na Tabela 2. A pesquisa com dados primários fundamenta-se na extração e análise de dados coletados diretamente pelos próprios participantes $^{21}$. Neste tipo de pesquisa os dados são sempre atualizados, uma vez que são coletados durante a realização do TCC, e são diretamente relacionados com a pesquisa, pois a coleta é elaborada pelo próprio pesquisador, baseado no que deve ser estudado. Já a pesquisa com dados secundários é realizada a partir de documentos e artefatos disponibilizados por instituições ou banco de dados já consolidados. Apesar da economia de tempo e dinheiro estes dados raramente se ajustam perfeitamente as necessidades da pesquisa presente, podendo estar desatualizados ou mesmo serem irrelevantes ${ }^{22}$.

\section{Tipo de estudo}

Quanto ao desenho do estudo houve quatro tipos de estudos abordados. A maioria dos estudos foi do tipo seccional (74\%), seguido por estudos do tipo ensaio clínico (18\%), estudos de coorte (5\%) e apenas um estudo do tipo caso-controle (3\%).

$\mathrm{O}$ estudo seccional caracteriza-se pela observação direta de uma determinada quantidade planejada de indivíduos em uma única oportunidade, por isso, necessita de um menor tempo de trabalho ${ }^{23}$. Possivelmente, estas características levaram os graduandos a desenvolverem a maioria dos TCC baseado neste tipo de estudo no período analisado.

No ensaio clínico o pesquisador provoca

Tabela 2 - Cenários dos TCC de acordo com os tipos de fonte para coleta de dados dos trabalhos do Curso de Odontologia nos anos de 2011 a 2013.

\begin{tabular}{lllllll}
\hline \multirow{2}{*}{ Cenário } & \multicolumn{2}{c}{ Dados primários } & \multicolumn{2}{c}{ Dados secundários } & \multicolumn{2}{c}{ TOTAL } \\
\cline { 2 - 6 } & $\mathrm{N}$ & $\%$ & $\mathrm{~N}$ & $\%$ & $\mathrm{~N}$ & $\%$ \\
\hline Clínica & 16 & 42 & - & 0 & 16 & 42 \\
Laboratório & 4 & 10 & 5 & 14 & 9 & 24 \\
Serviços de saúde & 6 & 16 & - & 0 & 6 & 16 \\
Outros & 5 & 14 & 2 & 5 & 7 & 18 \\
\cline { 1 - 4 } Total & \multirow{2}{*}{31} & 82 & 7 & 18 & 38 & 100 \\
\hline
\end{tabular}

uma intervenção intencional em algum aspecto do estado saúde do indivíduo, por meio da introdução de um esquema profilático ou terapêutico. Por se tratar de um estudo de intervenção este também sofrerá com maiores exigências do comitê de ética, o que pode dificultar a escolha do aluno por este tipo de trabalho, uma vez que o tempo para o desenvolvimento do TCC é restrito e ainda tem que se trabalhar com a espera da resposta do 
comitê.

Já os estudos do tipo coorte e casocontrole investigam a probabilidade que os indivíduos expostos a certos fatores adquiram subsequentemente uma determinada doença. Estes estudos são complexos e os resultados, geralmente, dependem de um acompanhamento ao longo do tempo ${ }^{24}$, o que dificulta a execução de um trabalho deste tipo durante a graduação.

Levando em consideração a referência temporal, foram realizados estudos do tipo transversal e estudos do tipo longitudinal. Nos estudos do tipo transversal todas as medições são realizadas em uma única ocasião ou durante um curto período de tempo, dessa forma, mostra-se o tipo de estudo mais condizente com o tempo que os alunos têm para o desenvolvimento dos $\mathrm{TCC}^{24}$.

Além disso, os estudos do tipo transversal são estudos de baixo custo, simplicidade analítica, alto potencial descritivo e rapidez na coleta acompanhada de facilidade na representatividade de uma população ${ }^{25}$. Já os estudos do tipo longitudinal estudam um fator ao longo do tempo para investigar mudanças, ou seja, refletem uma sequência de fatos ${ }^{26}$.

Apesar de geralmente os estudos longitudinais abordarem um menor número de sujeitos da pesquisa, o tempo necessário para a sua condução e os custos decorrentes disto torna-os estudos complexos ${ }^{27}$. Considerando estes elementos, conclui-se o motivo do estudo do tipo transversal ser mais utilizado nos projetos de pesquisa é o fato deste ser mais coerente com o tempo disponível para o desenvolvimento dos TCC.

\section{Comitê de Ética em Pesquisa (CEP)}

Do total de projetos analisados, 66\% previam a submissão ao CEP. Pela natureza do curso, é muito conveniente que haja pesquisas com seres humanos e, devido a isso, é necessária este apreciação. Vale salientar que não foi identificado nenhum projeto que utilizasse animais.

Em relação a este procedimento, a regulamentação federal visa garantir que a pesquisa com seres humanos seja conduzida de maneira eticamente aceitável ${ }^{28}$; dessa forma, pesquisas que envolvem seres humanos devem ser submetidas ao CEP para que, antes do início da coleta de dados, seja realizada a aprovação do projeto, evitando constrangimentos, despesas e riscos por parte dos sujeitos da pesquisa.

Possivelmente, o tempo de espera pela apreciação do CEP seria um fator desencorajador para os alunos durante $\mathrm{o}$ desenvolvimento do TCC, contudo, como mais de $50 \%$ dos trabalhos de propuseram a trabalhar com seres humanos pressupõe-se que as pesquisas estão sendo bem planejadas, prevendo em seu cronograma este tempo de espera.

\section{Referências bibliográficas}

As referências bibliográficas são os elementos fundamentais da pesquisa, a partir dos quais se obtêm as informações necessárias para o seu desenvolvimento. Após a análise observou-se referências retiradas de artigos, livros, teses e dissertações, sites ou publicações oficiais, conforme quantitativo apresentado no gráfico 1 .

Observou-se que das referências analisadas, $81 \%$ eram artigos, $11 \%$ livros, $3 \%$ teses e dissertações e 5\% outros documentos, em especial de sites ou publicações oficiais. Este é um resultado bastante significativo uma vez os artigos científicos configuram-se como fonte atualizada de informações.

$\mathrm{O}$ acesso aos artigos tem sido facilitado, o que pode ter contribuído para a maior parte das consultas referenciadas, uma vez que os processos de trabalho envolvidos na geração de conhecimento e produção acadêmica não são atividades triviais, pois demandam atualização constante $^{29}$. 
A produção científica dos TCC é uma conquista fundamental para estimular a criatividade, a educação permanente e a busca dos alunos de graduação por conhecimento. Sua inserção é um ótimo referencial para desenvolvimento da iniciação científica, a qual contribui para o aguçamento da visão crítica e a formação do aluno, além de refinar a capacida-

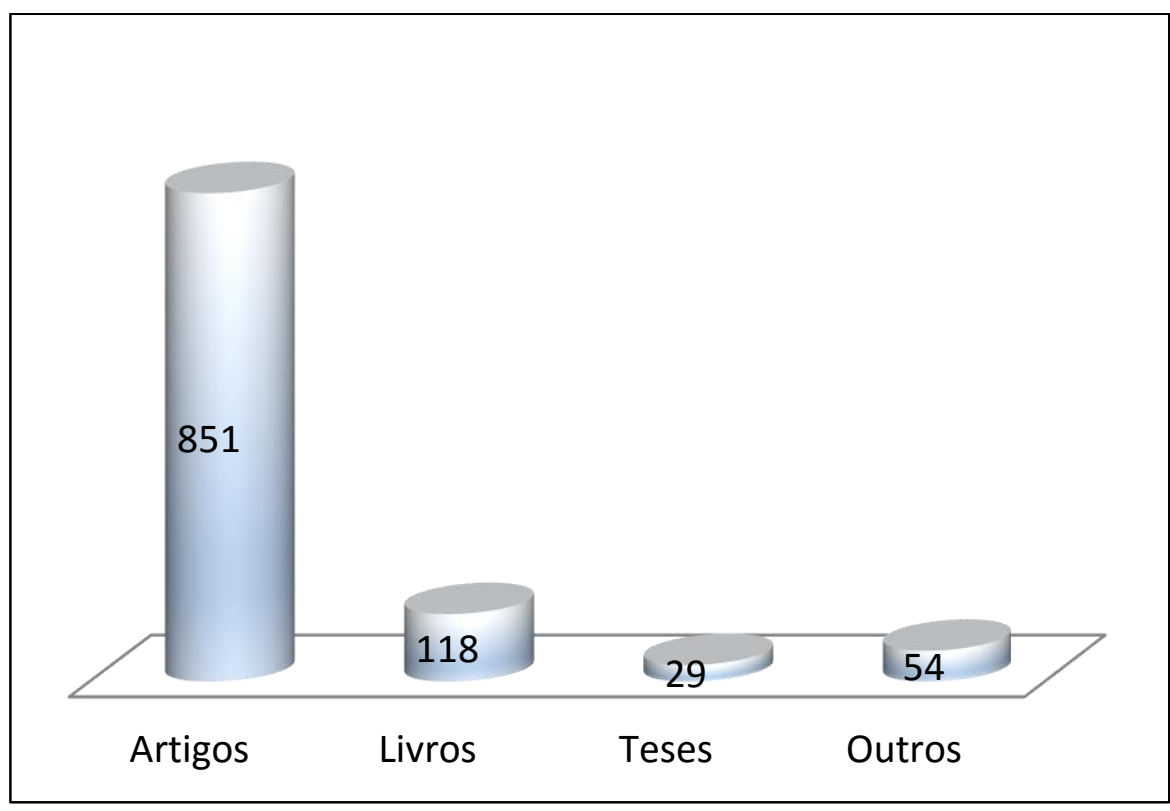

Gráfico 1 - Total de referências bibliográficas utilizadas nos TCC de Odontologia da UFRN, 2011 a 2013.

de de trabalhar com orientação e em grupo.

Os TCC devem ser entendidos não meramente como uma "tarefa" ao final do curso, mas um mecanismo efetivo para que a pesquisa seja compreendida como instrumento de aprendizado crítico pelo aluno, buscando relação de seus conhecimentos com as necessidades de saúde da população.

Seria importante enfatizar a construção de projetos de pesquisa coerentes com os princípios das DCN dos cursos de graduação em Odontologia, em especial com estudos que pudessem se comprometer com avanços no Sistema Único de Saúde.

Da mesma forma, seria oportuno que instituições que desenvolvem cursos de pósgraduação stricto sensu alimentassem suas linhas de pesquisa a partir destas pesquisas, o que permitiria um maior comprometimento do aluno de graduação com a construção do conhecimento e permitisse seu ingresso nestes programas com diferenciada possibilidade de inserção institucional.

\section{CONCLUSÕES}

O perfil da produção científica do curso de odontologia da UFRN realizada para o TCC é majoritariamente de estudos seccionais sobre temas de áreas clínicas, com coleta de dados primários, os quais são obtidos nas próprias dependências do curso. A maioria dos projetos é submetida ao CEP e utiliza artigos científicos como referências.

\section{REFERÊNCIAS}

1. Finkler M, Caetano JC, Ramos FRS. A dimensão ética da formação profissional em saúde: estudo de caso com cursos de graduação em odontologia. Ciênc Saúde Coletiva 2011;16(11):4481-92

2. Casotti E, Ribeiro VMB, Gouvea MV. Educação em odontologia no Brasil: produção de conhecimento no período 
1995-2006. História, Ciências, Saúde 2009;16(4):999-1010.

3. Demo P. Educar pela Pesquisa. $8^{\text {a }}$ ed. Campinas: Editores Associados, 2003

4. Guedes HTV, Guedes JC. Avaliação, pelos estudantes, da atividade "Trabalho de Conclusão de Curso" como integralização do eixo curricular de iniciação à pesquisa científica em um curso de Medicina. Rev Bras Educ Med. 2012; 36(2):162-71.

5. Brasil. Conselho Nacional de Educação. Câmara de Educação Superior. Diretrizes curriculares nacionais do curso de Graduação em Odontologia. Diário Oficial da União, Brasília, seção 1, p. 10, 4 mar. 2002.

6. Heyden MST, Resck ZMR, Gradim CVC. A pesquisa na graduação em enfermagem: requisito para conclusão do curso. Rev Bras Enferm 2003;56(4):409-11.

7. Cartes-Velasquez R, Aravena-Torres P. Perfil bibliométrico de la odontológia chilena, 2001-2010. Rev Clin Periodoncia Implantol Rehabil. Oral 2012;5(1):5-8.

8. Cury JA. The evolution of dental research in Brazil. Braz Oral Res. 2004;18(2):97.

9. Dias AA, Narvai PC, Rego DM. Tendências da produção científica em odontologia no Brasil. Pan Am J Public Health 2008;24(1):54-60.

10. Amorim KPC, Alves MSCF, Germano RM. A construção do conhecimento na Odontologia: a produção científica em debate. Acta Cir Bras. 2005;20(suppl1):811.

11. Vanti NAP. Da bibliometria à Webometria: uma exploração conceitual dos mecanismos utilizados para medir o registro da informação e a difusão do conhecimento. Ci Inf 2002;31(2):152-62.

12. Araújo CA. Bibliometria: evolução história e questões atuais. Em Questão 2006; 12(1):11-32.

13. Saupe R, Wendhausen ALP, Machado HB. Modelo para a implantação ou revitalização dos trabalhos de conclusão de curso. Rev Latino-am Enfermagem 2004;12(1):109-14.

14. Braccialli LAD, Oliveira MAC. Concepções de avaliação de desempenho em um currículo orientado por competência. Rev Esc Enferm USP 2011,45(5):1221-8.

15. Lemos M, Bazzo LMF. Formação do fonoaudiólogo no município de Salvador e consolidação do SUS. Ciênc. Saúde Coletiva 2010;15(5):2563-68.

16. Morita MC, Kriger L. Mudanças nos cursos de Odontologia e a interação com o SUS. Rev ABENO 2004;4(1):17-21.

17. Silveira JLGC. Diretrizes curriculares nacionais para os cursos de Graduação em odontologia: historicidade, legalidade e legitimidade. Pesq Bras Odontoped Clin Integr 2004;4(2):151-56.

18. Freitas SFT, Calvo MCM, Lacerda JT. Saúde coletiva e novas diretrizes curriculares em odontologia: uma proposta para graduação. Trab Educ Saúde 2012;10(2):223-34.

19. Noro LRA, Torquato SM. Percepção sobre o aprendizado de saúde coletiva e o SUS entre alunos concludentes de curso de odontologia. Trab Educ Saúde 2011;8(3) :439-447.

20. Fadel CB, Baldani MH. Percepções de formandos do curso de odontologia sobre as Diretrizes Curriculares Nacionais. Trab Educ Saúde 2013;11(2):339-54.

21. Kovacs MH, Leão ALMS, Vieira RG, Barbosa L, Dias CM. Podemos confiar nos resultados de nossas pesquisas? Uma avaliação dos procedimentos metodológicos nos artigos de marketing do EnANPAD. Encontro de Marketing da ANPAD, I EMA, 2004. Anais.

22. Grady D, Hearst N. Usando bases de dados existentes. In: Hulley SB (org). Delineando a pesquisa clínica. Uma abordagem epidemiológica. Porto Alegre: Artmed. 2008. p. 225-39.

23. Klein $\mathrm{CH}$, Bloch KV. Estudos seccionais. IN: Medronho RA, Bloch K. Epidemiologia. $2^{a}$ ed. São Paulo: Atheneu, 2008. p.193-219.

24. Newman T, Cummings S, Hulley SB. Delineando estudos de coorte. In: Hulley SB (org). Delineando a pesquisa clínica. Uma abordagem epidemiológica. Porto Alegre : Artmed, 2008. p. 115-25.

25. Sitta EI, Arakawa AM, Caldana ML, Peres SHCS. A contribuição de estudos 
transversais na área da linguagem com enfoque em afasia. Rev CEFAC 2010;12(6):1059-66.

26. Hochman B, Xerfan FN, Oliveira Filho RS, Ferreira LM. Desenhos de pesquisa. Acta Cir Bras. 2005;20(2):2-9.

27. Mota MMPE. Metodologia da pesquisa em desenvolvimento humano: velhas questões revisitadas. Psicologia em Pesquisa 2010;4(2):144-49.

28. Lo B. Delineando estudos transversais e de caso-controle. In: HULLEY Stephen (org). Delineando a pesquisa clínica. Uma abordagem epidemiológica. Ed: Artmed, 2008. p. 243-56.

29. Dudziak EA, Villela COM, Barbin SE. Impacto da Internet e das bibliotecas digitais na pesquisa científica: o caso da Engenharia Elétrica na Escola Politécnica da USP. In: Simpósio Internacional de Bibliotecas Digitais, 3, 2005, São Paulo. SIDB: trabalhos. São Paulo: CRUESP/ ISTEC, 2005.

\footnotetext{
ABSTRACT

Bibliometric analysis of undergraduate final work in a Northeast Brazilian Dental School The aim of the study was to identify trends in scientific production of the dentistry course, by Undergraduate Final Work (Trabalho de Conclusão de Curso - TCC), which are the development of a research by undergraduate student, based on the need of knowledge production and continuing education. It is a bibliometric research through cross-sectional, observational descriptive study with a quantitative approach. It was observed predominance of cross-sectional studies, developed with humans, focused on clinical area and $82 \%$ performed with primary data. Of
}

the total, $62 \%$ were submitted to the ethics committee in research. It was observed that $81 \%$ of the references cited were journal articles, $11 \%$ books, $3 \%$ theses and dissertations, and 5\% other documents, in particular websites or official publications. The TCC must be understood as fundamental to stimulate creativity achievement, lifelong learning and the pursuit of knowledge by students from the research and not merely a task required by the Pedagogic Project. It would be also important to emphasize the construction of projects consistent with the principles of the National Curriculum Guidelines of Dentistry courses, particularly with studies to commit the advances in the Unified Health System research. The profile of scientific production from UFRN dental course conducted for TCC is mostly sectional studies on clinical area, with primary data collection, which are obtained in own premises of the course. Most of the projects are submitted to the CEP and uses scientific articles as references.

Descriptors: Bibliometrics. Scientific Publication Indicators. Dentistry.

Correspondência para:

Prof. Dr. Luiz Roberto Augusto Noro

e-mail: luiz_noro@hotmail.com

Departamento de Odontologia da UFRN

Avenida Salgado Filho, 1787

59056-000 - Natal/RN 